\title{
As Relações de Vinculação e a Imagem Corporal: Exploração de um Modelo ${ }^{1}$
}

\author{
Maria Raquel Barbosa ${ }^{2}$ \\ Paula Mena Matos \\ Maria Emília Costa \\ Universidade do Porto
}

\begin{abstract}
RESUMO - Pretende-se analisar a contribuição diferencial dos principais contextos de desenvolvimento (relação parental e de pares) na forma como os adolescentes experienciam a relação com o seu corpo. A amostra é constituída por 690 adolescentes e jovens adultos (242 meninos e 448 meninas), com idades compreendidas entre os 15 e os 23 anos $(M=17,8$ e $D P=2,00)$. Os resultados do modelo de predição revelaram que o gênero e a vinculação romântica foram as variáveis que mais contribuíram para a variação da variável imagem corporal. Foi observado um efeito de mediação entre a vinculação aos pais e a imagem corporal através da relação romântica e de amizade. Os resultados sublinham a necessidade de se compreender a vivência corporal nos contextos interpessoais.
\end{abstract}

Palavras-chave: imagem corporal, vinculação, adolescência, modelo preditivo.

\section{Attachments and Body Image: An Exploratory Model}

\begin{abstract}
The present study explored the differential effects of the principal development contexts (attachment to parents and peers) on the way in which adolescents experience their bodies. Six hundred and ninety adolescents and young adults (242 male and 448 female) participated in this study, with ages varying between 15 and 23 years $(M=17,8$ and $S D=2,00)$. The results of the prediction model revealed that gender and romantic attachment were the variables that showed the strongest effect on the variation in body image. A mediation effect was observed among attachment to parents and body image through romantic and friendship relationships. The results highlight the need of understanding body experience in an interpersonal context.
\end{abstract}

Key- words: body image, attachment, adolescence, predictive model.

Podemos dizer com uma grande certeza que a imagem corporal é um aspecto central no desenvolvimento psicológico e interpessoal na adolescência e que tem se tornado alvo de atenção preferencial nas pesquisas. As contradições e as dificuldades no estudo da imagem corporal apontam para a necessidade de ampliar a nossa compreensão sobre o tema. A imagem do corpo condensa o "conjunto de representações, sentimentos, atitudes que o indivíduo elaborou acerca do seu próprio corpo ao longo da existência" (Bruchon-Schweitzer, 1990, p. 173-174), através de experiências não apenas sensoriais e cognitivas, mas também afetivas e sociais (Cash \& Pruzinsky, 1990, 2004). Será, assim, uma construção biopsicossocial, parcialmente determinada por, mas não reduzida a um corpo físico e objetivo, salientando-se a vivência dinâmica e emocional de um corpo imbuído de significados, construídos ao longo do tempo e baseados nas experiências vividas.

Embora a investigação esteja atenta ao fato dos corpos terem se tornado irrealistamente magros nos ideais culturais, tem havido pouca ênfase em entender o mecanismo pelo

1 Apoio: Este estudo está incluído num Projecto financiado pela Fundação para a Ciência e Tecnologia (Projecto POCI/ PSI/ 61722/ 2004).

2 Endereço para correspondência: Faculdade de Psicologia e de Ciências da Educação da UP. Rua Dr. Manuel Pereira da Silva. Porto, Portugal. Fone: 00351 226079779; Fax: 00351 226079727.E-mail: raquel@ fpce.up.pt qual as pressões sociais para alcançar a figura ideal afetam a imagem e experiência corporais. Alguns autores começam a preocupar-se com as "influências sub-culturais mais imediatas" (Benedikt, Wertheim \& Love, 1998) como os pais, pares e pares românticos, acreditando que estes contextos basilares de desenvolvimento desempenham um papel essencial no desenvolvimento da imagem corporal (Cash \& Fleming, 2004; Kearney-Cooke, 2004; McKinley \& Randa, 2005; Tantleff-Dunn \& Gokee, 2004).

As relações entre a vinculação parental e o corpo têm sido objeto de alguns estudos. Na revisão de literatura encontrada, o primeiro estudo que relaciona a vinculação parental e as perturbações do comportamento alimentar foi conduzido por Armstrong e Roth (1989). Os autores avaliaram 27 doentes (11 anoréticas, 12 bulímicas e quatro casos atípicos de perturbações alimentares) e observaram que $96 \%$ das participantes foram classificadas com uma vinculação ansiosa. Por outro lado, Sharpe et al. (1998) encontraram uma associação entre o estilo de vinculação aos pais e as preocupações com o corpo em adolescentes do gênero feminino (dos nove aos 12 anos), considerando esta relação um fator de risco para o desenvolvimento de perturbações do comportamento alimentar. Outros autores corroboraram estes resultados salientando que um padrão de vinculação inseguro, particularmente o estilo preocupado ou ansioso, tem sido encontrado nas populações com preocupações exacerbadas com o corpo 
(Broberg, Hjalmers \& Nevonen, 2001, Suldo \& Sandberg, 2000; Ward, Ramsay \& Treasure, 2000).

Adicionalmente, uma imagem corporal mais positiva tem sido predita por relações parentais positivas e pouco conflituosas (Archibald, Graber \& Brooks-Gunn, 1999; Barbosa, 2001). Apesar de se começar a considerar a importância da figura paterna na investigação, poucos estudos têm incluído o impacto das relações com o pai na construção da imagem corporal. Domini, Johnson e Koch (2000), num estudo com mulheres, referem que aquelas que são diagnosticadas com perturbações alimentares percebem o seu pai como mais rejeitadores do que as mulheres do grupo controle. Num outro estudo com 400 adolescentes portugueses (dos 15 aos 20 anos de idade) foi encontrado um efeito significativo dos estilos de vinculação ao pai na imagem corporal, sendo os seguros mais satisfeitos com o peso e menos centrados na aparência física do que os preocupados e amedrontados; os desinvestidos, tal como os seguros, menos preocupados com a aparência do que os preocupados e amedrontados e os amedrontados mais preocupados com a aparência física que os preocupados (Barbosa, 2001).

A adolescência é um período de intensa sociabilidade, em que as normas vigentes no grupo de pares e as expectativas sociais têm uma importância acrescida no desenvolvimento. É comum o adolescente imitar os pares, adotando hábitos, atitudes e indumentárias do seu grupo etário, já que a proximidade do modelo é tranquilizadora. Com efeito, a influência da aceitação social, das relações e a popularidade no grupo de pares, contribui para a avaliação do self do adolescente.

Pesquisas têm sugerido que as relações de amizade e o grupo de pares providenciam uma subcultura que enfatiza a importância da magreza, através de dietas, da pressão do grupo acerca do peso e forma corporais (Lieberman, Gauvin, Bukowski \& White, 2001; Lunner, Wertheim, Thompson, Paxton, McDonald \& Halvarsson, 2000).

Menos estudos se têm centrado na influência da qualidade das relações de amizade nos comportamentos e preocupação com a imagem corporal. Alguns têm encontrado uma associação entre uma relação de pares que fornece pouco apoio e a insatisfação corporal em adolescentes do gênero feminino (Gerner \& Wilson, 2005; Stice \& Whitenton, 2002). Outros evidenciaram uma associação entre mulheres com problemas de imagem corporal e dificuldades em estabelecerem relações de intimidade (Evans \& Wertheim, 2005) ou vinculações inseguras com os pares (Okine, 2000).

Um dos menos explorados, mas talvez o fator interpessoal mais importante na construção da imagem corporal nesta etapa de desenvolvimento é o papel do par amoroso e da qualidade desta relação. Os adolescentes estão particularmente preocupados com a sua aparência física, pois é nesta altura que começam a ter relacionamentos com o sexo oposto, o que aumenta o contato físico e, portanto, a exposição do seu corpo à avaliação dos outros, podendo ser um fator importante na construção e satisfação numa relação romântica (O’Dea \& Abraham, 1999). Um obstáculo frequentemente apontado para se iniciar uma relação é a falta de confiança na sua aparência física, na sua imagem corporal, e o fato de que as pessoas que se sentem fisicamente não atraentes são abordadas menos vezes pelos outros no sentido de começarem um relacionamento (Sprecher, McKinney \& Orbuch,
1991). Assim, o par amoroso será uma importante fonte de influência através do feedback que transmite acerca da imagem ou aparência do outro, podendo estar mais preocupado em conhecer os ideais do outro e não os ideais estabelecidos pela sociedade em geral.

Num estudo recente, Cash, Theriault e Annis (2003) exploraram tanto a vinculação adulta em geral como a vinculação romântica em jovens estudantes e encontraram uma relação positiva entre a vinculação segura e a satisfação corporal; na vinculação romântica, uma maior ansiedade estava relacionada com uma menor satisfação com o corpo. Estes resultados são consistentes com o estudo de Brennan e Shaver (1995), que encontraram medidas de insatisfação corporal associadas a um estilo de vinculação preocupado. Uma explicação para este fato poderá ser que, aqueles que evidenciam níveis mais elevados de ansiedade nas suas relações de vinculação estão mais atentos a determinados fenômenos sociais valorizados por muitos, como a aparência, enquanto a evitação da proximidade poderá indicar uma pessoa que se rege maioritariamente pelos seus próprios padrões físicos (McKinley \& Randa, 2005).

$\mathrm{O}$ gênero tem sido reconhecido como um fator saliente no desenvolvimento da imagem corporal. É geralmente aceito que as adolescentes do gênero feminino tendem a ser mais preocupadas e insatisfeitas com o seu corpo do que os rapazes, investindo mais cognitiva e comportamentalmente na sua aparência física (Demarest \& Allen, 2000; Gaspar, 1999). Muitos investigadores e feministas têm afirmado que as normas e expectativas sociais encorajam as mulheres a estarem atentas e psicologicamente investidas na sua aparência física, o que pode desestabilizar o seu bem-estar e contribuir para o desregular da alimentação, para a depressão ou outras dificuldades psicológicas (Cash \& Pruzinsky, 1990; Thompson, 1996). De fato, relativamente ao gênero masculino, as mulheres investem mais na sua aparência (Muth \& Cash, 1997).

No entanto, as preocupações com a imagem corporal, a insatisfação e preocupação com a aparência também começam a afetar os homens (Barbosa, 2001; Cash \& Pruzinsky, 1990; McCabe \& Ricciardelli, 2001; Ricciardelli, McCabe \& Banfield, 2000). Claramente, a investigação tem sugerido que a imagem corporal é um problema tanto de mulheres como de homens. Contudo, enquanto a maioria das mulheres deseja perder peso, os homens parecem estar divididos entre aqueles que querem perder peso e aqueles que pretendem ganhar peso, entendido como ganhar massa muscular (McCabe \& Ricciardelli, 2001).

Contudo, se o gênero tem sido apontado como uma variável diferenciadora, a idade já não segue um padrão de resultados tão consonante, havendo estudos que apontam para diferenças etárias em ambos os sexos (Green \& Pritchard, 2003) e outros afirmando que as pressões para um corpo "ideal" parecem abranger todas as faixas etárias (Demarest \& Allen, 2000; Grogan, 1999), demonstrando que a insatisfação corporal começa desde cedo e acompanha os indivíduos ao longo de seu desenvolvimento, sendo mais saliente quando das transformações físicas que caracterizam a puberdade.

Em suma, a premissa fundamental de que o nosso corpo é um corpo relacional, que apenas se constrói e desenvolve na relação com os outros, tornou-se a pedra basilar desta 
investigação. Assim, este estudo, focalizado numa população não-clínica, pretende explorar a relação entre a qualidade das relações de vinculação do adolescente e a imagem corporal nos gêneros masculino e feminino. Particularmente, pretende-se avaliar quais os principais preditores da imagem corporal incluindo os três componentes da imagem corporal - avaliação, investimento e afeto (Cash, 2004; Cash \& Prusinsky, 2004), tendo em conta a vinculação aos pais, namorado(a) e amigos, assim como a idade e o gênero em adolescentes portugueses, a partir das seguintes questões de investigação: 1 - Sabendo que a adolescência é uma fase de desenvolvimento caracterizada por transformações e mudanças físicas, psicológicas e relacionais, quais serão, entre as relações parentais, amorosas ou de amizade, os melhores preditores interpessoais da imagem corporal? 2 - Serão as relações com os pares (romântica e de amizade) mediadoras entre a qualidade da relação parental e a satisfação com a imagem corporal? 3 - Considerando o gênero como uma importante variável relacional e corporal, qual será o seu efeito em cada uma das variáveis presentes no modelo? 4 - Será que a imagem corporal e a qualidade das relações interpessoais variam com a idade?

\section{Método}

\section{Participantes}

A amostra deste estudo é constituída por 690 participantes: 242 do sexo masculino e 448 do sexo feminino. A idade varia entre os 15 e os 23 anos $(M=17,8$ e $D P=2,00)$. Os sujeitos frequentavam diversas escolas secundárias e faculdades públicas do grande Porto. Apenas foram selecionados os participantes pertencentes a famílias intactas - mãe, pai, irmão(s) e/ou avó(ô) - e que mantivessem (50,3\%) ou já tivessem tido $(49,7 \%)$ uma relação romântica. Relativamente à duração da relação, $37,5 \%$ atingiram uma relação romântica de seis meses, $20,3 \%$ entre seis meses e um ano; $17,4 \%$ uma relação de um a dois anos e 14,8\% atingiram um relacionamento com mais de dois anos de duração.

Os instrumentos foram administrados, de forma aleatória em termos sequenciais (contra-balanceamento), a grupos-turma, sob supervisão do professor da classe e investigador principal, de acordo com instruções padronizadas. Foi assegurada a confidencialidade e o anonimato das respostas e a natureza voluntária da participação de cada adolescente.

\section{Instrumentos}

Inventário de Vinculação aos pais e pares (IPPA; Armsden \& Greenberg, 1987) é uma medida de auto-relato constituída por uma subescala de 28 itens, que avalia a qualidade da relação parental e por 25 itens que avaliam a vinculação aos pares (a subescala utilizada neste estudo). Os itens organizam-se em três dimensões: Confiança $(\alpha=0,95)$, Comunicação $(\alpha=0,92)$, avaliada na sua extensão e qualidade e Alienação $(\alpha=0,65)$ que descreve sentimentos de alienação e isolamento, mas também o reconhecimento da necessidade de ter amigos próximos. Os alphas apresentados são relativos à amostra deste estudo.

Questionário de Vinculação Amorosa (QVA; Matos, Barbosa \& Costa, 1998, 1999) é um instrumento de auto-relato desenvolvido para a população portuguesa. É constituído por 56 itens e pretende avaliar as percepções dos adolescentes acerca da vinculação ao seu parceiro amoroso. O questionário avalia quatro dimensões: Confiança na acessibilidade da figura de vinculação; Dependência $(\alpha=0,87)$, refere-se à necessidade emocional e física de proximidade, ao medo da perda; Evitamento $(\alpha=0,86)$ traduz o papel secundário do par romântico relativamente às necessidades de vinculação $\mathrm{e}$ Ambivalência $(\alpha=0,74)$ revela a incerteza relativamente ao papel do par romântico e às emoções na relação.

Questionário de Vinculação ao Pai e à Mãe (QVPM; Matos, Almeida \& Costa, 1998). Este instrumento de auto-relato, desenvolvido também para a população portuguesa, é constituído por 44 itens e avalia as percepções dos adolescentes e jovens adultos acerca das relações de vinculação com cada uma das figuras parentais. É baseado numa abordagem dimensional da vinculação adulta, nomeadamente no modelo de Kim Bartholomew (Bartholomew, 1990; Bartholomew \& Horowitz, 1991) e é constituído por três dimensões para a vinculação ao pai e à mãe: Inibição da Exploração e Individualidade $\left(\alpha_{\text {pai }}=0,88 ; \alpha_{\text {mãe }}=0,87\right)$ que avalia a percepção dos sujeitos acerca da inibição parental da autonomia e diferenciação; Qualidade do Laço Emocional $\left(\alpha_{\text {pai }}=0,83 ; \alpha_{\text {mãe }}=0,79\right)$, relativo à qualidade da proximidade emocional aos pais e a Ansiedade de Separação $\left(\alpha_{\text {pai }}=0,68\right.$; $\left.\alpha_{\text {mãe }}=0,66\right)$ revela a necessidade de proximidade, ansiedade de separação e medo da perda.

Escala de Estima Corporal (EEC, Franzoi \& Shields, 1984; versão portuguesa, Barbosa, 2001): este questionário enumera 35 aspectos da aparência e funcionamento físicos. Foram encontrados três fatores: Satisfação com o peso $(\alpha=$ $0,94)$ diz respeito à aparência física, mas inclui as partes do corpo que podem ser fisicamente alteradas através do exercício ou controle alimentar; a Atractividade física $(\alpha=0,83)$, relacionada com as feições faciais ou outros aspectos que podem ser modificados através de cosméticos; e a Satisfação com a sexualidade $(\alpha=0,78)$ associada às partes corporais ligadas à sexualidade.

Inventário de Esquemas de Aparência (IEA; Cash \& Labarge, 1996 - versão portuguesa, Barbosa, 2001): é uma escala composta por 14 itens construída para avaliar crenças ou convicções acerca da importância, significado e efeitos da aparência na vida de uma pessoa. Os itens organizam-se num fator denominado Preocupação com a aparência $(\alpha=0,78)$ que envolve a predisposição para sentir a aparência como um aspecto central para o auto-conceito e engloba pressupostos acerca do bom/mau de uma pessoa ser atraente/não atraente.

Inventário de Perturbações Alimentares (EDI-2; Garner, 1991). Embora o estudo das perturbações alimentares não seja um objetivo deste estudo, consideramos pertinente o uso deste instrumento devido à diversidade de dimensões da imagem corporal abordadas. EDI-2 é um questionário de auto-relato, composto por 64 itens, com 27 itens adicionais, que formam três novos construtos da escala. As dimensões do EDI são: Desejo de magreza, Bulimia, Insatisfação corporal, Ineficácia, Perfeccionismo, Desconfiança interpessoal, Cons- 
ciência interoceptiva, Asceticismo, Regulação dos impulsos, Insegurança social e Medo da maturidade com alphas entre 0,69 e 0,90 .

\section{Resultados}

Antes de se proceder às análises estatísticas, a base de dados foi avaliada quanto à existência de valores omissos, outliers, normalidade multivariada e multicolinearidade. Os softwares estatísticos utilizados para as análises foram o SPSS (versão 15.0) e o EQS 6.1 para Windows.

As 15 variáveis dependentes, obtidas pelos questionários acima mencionados foram submetidas a uma análise exploratória em componentes principais, visando-se a redução do número de variáveis e, consequentemente um modelo mais parcimonioso.

Analisando-se a estrutura fatorial obtida, verificou-se que deviam ser eliminadas as variáveis Medo da maturidade e Consciência interoceptiva por saturarem em mais do que um fator e devido aos baixos valores de comunalidade (no caso do Medo da maturidade). Obteve-se, assim, uma estrutura fatorial composta por 13 variáveis (subescalas), distribuídas por quatro fatores que explicam $72 \%$ da variância total, sendo $38,9 \%$ da variância explicada pelo $1^{\circ}$ fator, $12,8 \%$ pelo $2^{\circ}, 12,4 \%$ pelo $3^{\circ}$ fator e $8,1 \%$ pelo $4^{\circ}$ e último fator. Os fatores encontrados, relacionados de uma forma mais ou menos direta com dimensões corporais, foram nomeados de: Desejo de magreza, Insegurança social, Estima corporal e Perfeccionismo (ver Tabela 1). A nomeação dos fatores teve em conta a contribuição de cada uma das variáveis, bem como um conceito que abarcasse, de uma forma geral, todas elas.
O primeiro fator agrupa quatro subescalas cujas saturações variam entre 0,68 e 0,88 . Estas aparecem relacionadas com a preocupação excessiva pelo peso corporal, pelo medo de ganhar peso, com dietas, com as partes do corpo que podem ser fisicamente alteradas através do exercício físico ou do controle da alimentação. Avalia ainda a tendência para a autodisciplina, o sacrifício e capacidade de se abster de prazeres corporais, mas também a tendência em pensar ou ter acessos de voracidade alimentar. Por tudo isto a designação de Desejo de magreza.

O segundo fator, Insegurança social, é composto por três subescalas e associa variáveis relacionadas com aspectos de natureza social e psíquica: com a crença de que as relações sociais são tensas, inseguras, uma desilusão; mede ainda o sentimento geral de alienação e relutância para formar relações próximas e o sentimento de descrédito por si mesmo (baixa auto-estima), de vazio e solidão. Uma vez que o objetivo do trabalho é avaliar a imagem corporal, optou-se por não considerar este fator como variável dependente nas análises subsequentes.

O terceiro fator, Estima corporal, é formado por três subescalas e o seu conteúdo reflete a percepção dos sujeitos acerca da estima que têm por cada uma das partes do seu corpo e pela sua sexualidade. Este fator agrupou as mesmas dimensões que definem a Escala de Estima Corporal (BES), utilizada neste estudo e acima descrita, o que reforça a tridimensionalidade do constructo "estima corporal".

Finalmente, a Centralidade da aparência e Perfeccionismo engloba as três últimas variáveis relativas à crença de que apenas os níveis mais elevados de desempenho pessoal são aceitáveis e que os outros só esperam isso de si próprio. Além de características perfeccionistas, este fator mede também a

Tabela 1. Estrutura factorial das variáveis da imagem corporal (Análise em Componentes Principais e com rotação "varimax"):

\begin{tabular}{|c|c|c|c|c|c|}
\hline Variáveis & $\begin{array}{c}\text { Factor } 1 \\
\text { (Desejo de } \\
\text { magreza) }\end{array}$ & $\begin{array}{c}\text { Factor } 2 \\
\text { (Insegurança } \\
\text { social) }\end{array}$ & $\begin{array}{c}\text { Factor } 3 \\
\text { (Estima } \\
\text { corporal) }\end{array}$ & $\begin{array}{c}\text { Factor } 4 \\
\text { (Perfeccionismo) }\end{array}$ & $h^{2}$ \\
\hline - Desejo de magreza & 0,881 & & & & 0,814 \\
\hline - Insatisfação corporal & 0,789 & & & & 0,708 \\
\hline - Asceticismo & 0,698 & & & & 0,726 \\
\hline - Bulimia & 0,684 & & & & 0,633 \\
\hline - Insegurança social & & 0,865 & & & 0,817 \\
\hline - Desconfiança interpessoal & & 0,846 & & & 0,732 \\
\hline - Ineficácia & & 0,740 & & & 0,760 \\
\hline - Atractividade física & & & 0,813 & & 0,751 \\
\hline - Satisfação com a sexualidade & & & 0,812 & & 0,708 \\
\hline - Satisfação com o peso & & & 0,719 & & 0,849 \\
\hline - Perfeccionismo & & & & 0,836 & 0,718 \\
\hline - Regulação dos impulsos & & & & 0,589 & 0,582 \\
\hline - Preocupação aparência & & & & 0,549 & 0,476 \\
\hline Valor próprio & 3,171 & 2,841 & 2,241 & 1,890 & \\
\hline \% Variância explicada & $38,9 \%$ & $12,8 \%$ & $12,4 \%$ & $8,1 \%$ & \\
\hline Variância total explicada & $72 \%$ & & & & \\
\hline
\end{tabular}


Tabela 2. Índices de ajustamento (robustos) para as AFC de segunda ordem, respeitantes às diferentes escalas e/ou constructos utilizados

\begin{tabular}{|c|c|c|c|c|c|c|c|}
\hline \multirow{2}{*}{ Escala } & \multirow{2}{*}{\multicolumn{3}{|c|}{ Dimensões das escalas }} & \multicolumn{4}{|c|}{ Índices } \\
\hline & & & & $\mathbf{S}-\mathbf{B} \chi^{2 b}$ & gl & $\mathbf{C F I}^{\mathrm{c}}$ & RMSEA $^{\mathrm{d}}$ \\
\hline Vinculação ao pai & IEIpai & QLEpai & ASDpai & \multirow{3}{*}{$772,7^{*}$} & \multirow{3}{*}{320} & \multirow{3}{*}{0,90} & \multirow{3}{*}{$\begin{array}{c}0,04 \\
(0,04,0,05)\end{array}$} \\
\hline Peso original & 0,85 & 0,89 & 0,31 & & & & \\
\hline Peso ponderado ${ }^{\mathrm{a}}$ & 0,42 & 0,43 & 0,15 & & & & \\
\hline Vinculação à mãe & IEImãe & QLEmãe & ASDmãe & \multirow{3}{*}{$731,0^{*}$} & \multirow{3}{*}{320} & \multirow{3}{*}{0,89} & \multirow{3}{*}{$\begin{array}{c}0,04 \\
(0,04,0,05)\end{array}$} \\
\hline Peso original & 0,86 & 0,88 & 0,25 & & & & \\
\hline Peso ponderado & 0,43 & 0,44 & 0,13 & & & & \\
\hline Vinculação aos amigos & COMUNICAÇÃO & CONFIANÇA & ALIENAÇÃO & \multirow{3}{*}{$587,1^{*}$} & \multirow{3}{*}{228} & \multirow{3}{*}{0,90} & \multirow{3}{*}{$\begin{array}{c}0,05 \\
(0,04,0,05)\end{array}$} \\
\hline Peso original & 0,92 & 0,95 & 0,65 & & & & \\
\hline Peso ponderado & 0,36 & 0,38 & 0,26 & & & & \\
\hline Vinculação romântica ${ }^{\mathrm{e}}$ & CONFIANÇA & EVITAMENTO & AMBIVALÊNCIA & \multirow{3}{*}{$454,5^{*}$} & \multirow{3}{*}{115} & \multirow{3}{*}{0,92} & \multirow{3}{*}{$\begin{array}{c}0,06 \\
(0,06,0,07)\end{array}$} \\
\hline Peso original & 0,89 & 0,92 & 0,78 & & & & \\
\hline Peso ponderado & 0,34 & 0,35 & 0,30 & & & & \\
\hline \multirow[t]{2}{*}{ Imagem Corporal $^{\mathrm{f}}$} & SATISFAÇÃO & ESTIMA & N_CENTRALIDADE & \multirow{4}{*}{$127,7 *$} & \multirow{4}{*}{21} & \multirow{4}{*}{0,94} & \multirow{4}{*}{$\begin{array}{c}0,09 \\
(0,07,0,1)\end{array}$} \\
\hline & PESO & CORPORAL & APARÊNCIA & & & & \\
\hline Peso original & 0,96 & 0,58 & 0,83 & & & & \\
\hline Peso ponderado & 0,40 & 0,24 & 0,35 & & & & \\
\hline
\end{tabular}

Nota: $* \mathrm{p}<0,01$

Legenda: IEI= Inibição da Exploração e Individualidade (para o pai e mãe); QLE= Qualidade de Laço Emocional; ASD=Ansiedade de Separação e Dependência.

a) A segunda linha para cada escala, são as ponderações proporcionais de cada coeficiente de regressão, totalizando a soma destes 1; b) Satorra-Bentler chi square; c) CFI= Comparative Fit Index; d) RMSEA= Root Mean Square Error of Approximation com o respectivo intervalo de confiança a 90\%; e) A subescala dependência foi eliminada, devido ao seu baixo valor de correlação com o factor de segunda ordem $(r=0.16)$; f) Esta variável compósita derivou da AFE acima referida. A dimensão Insatisfação social foi extraída do modelo.

Foram invertidas 3 subescalas para uma medida positiva de imagem corporal (desejo de magreza; insegurança social e perfeccionismo, para satisfação com o peso, segurança social e não perfeccionismo, respectivamente).

tendência para a impulsividade, para a hostilidade e destruição das relações interpessoais. Salienta ainda a centralidade que a aparência física pode ter na vida das pessoas.

Foram encontrados alphas de 0,82 para o Desejo de magreza, $\alpha=0,79$ para o fator Estima corporal e $\alpha=0,54$ para a Centralidade da aparência e Perfeccionismo. Estes valores indicam uma boa consistência interna para as duas primeiras variáveis e medíocre para o último fator $(\alpha=0,54)$. No entanto, apesar do valor baixo do alpha neste fator, decidiu-se mantê-lo nas análises subsequentes, uma vez que esta medida de consistência interna é bastante influenciada pelo número de itens de uma escala, apesar da variância de erro individual e partilhada pelos itens (Rowe, 2002).

Foram usadas análises fatoriais confirmatórias (AFC) para testar a validade das variáveis ou fatores de primeira ou segunda ordem. Estas estruturas fatoriais derivaram de análises fatoriais exploratórias prévias. O procedimento utilizado para a estimativa dos parâmetros foi o método da máxima verosimilhança (Maximum Likelihood) e a qualidade do ajustamento do modelo foi efetuada de forma global e local/individual. Os modelos propostos foram avaliados através da matriz de covariância. Um resumo dos índices de ajustamento global obtidos para cada uma das escalas está representado na Tabela 2 .
Como se pode verificar, todas as escalas/fatores analisados revelaram um ajustamento aceitável a esta amostra de adolescentes e jovens adultos, embora não perfeito. Relativamente à consistência interna de cada uma das dimensões/ indicadores, foram encontrados valores de alpha de Cronbach que vão desde 0,61 (na vinculação ao pai e à mãe), 0,89 na escala de vinculação romântica, 0,76 na relação com os amigos e 0,83 no constructo imagem corporal.

Após a obtenção de uma estrutura fatorial satisfatória para cada dimensão/indicador considerada, procedeu-se à computação de medidas compostas, com vista à obtenção de um modelo mais simplificado, com um menor número de variáveis e parâmetros a estimar. Estas medidas foram calculadas tendo em conta os pesos dos coeficientes de regressão (ponderados) de cada item para cada dimensão, obtidos na $\mathrm{AFC}^{3}$ (ver na tabela 2, na $2^{\mathrm{a}}$ linha de cada variável). Este procedimento minimiza o erro de medida dos itens que contribuem para cada escala, aumentando, portanto a consistência

3 Os pesos obtidos na AFC (iniciais) foram ponderados (proporcionalmente em função do seu peso relativo) para que a sua soma totalizasse 1 . Pretendeu-se com este procedimento assegurar que a medida composta obtida tivesse um limite mínimo e um máximo idêntico ao de cada item, e consequentemente a mesma amplitude. 


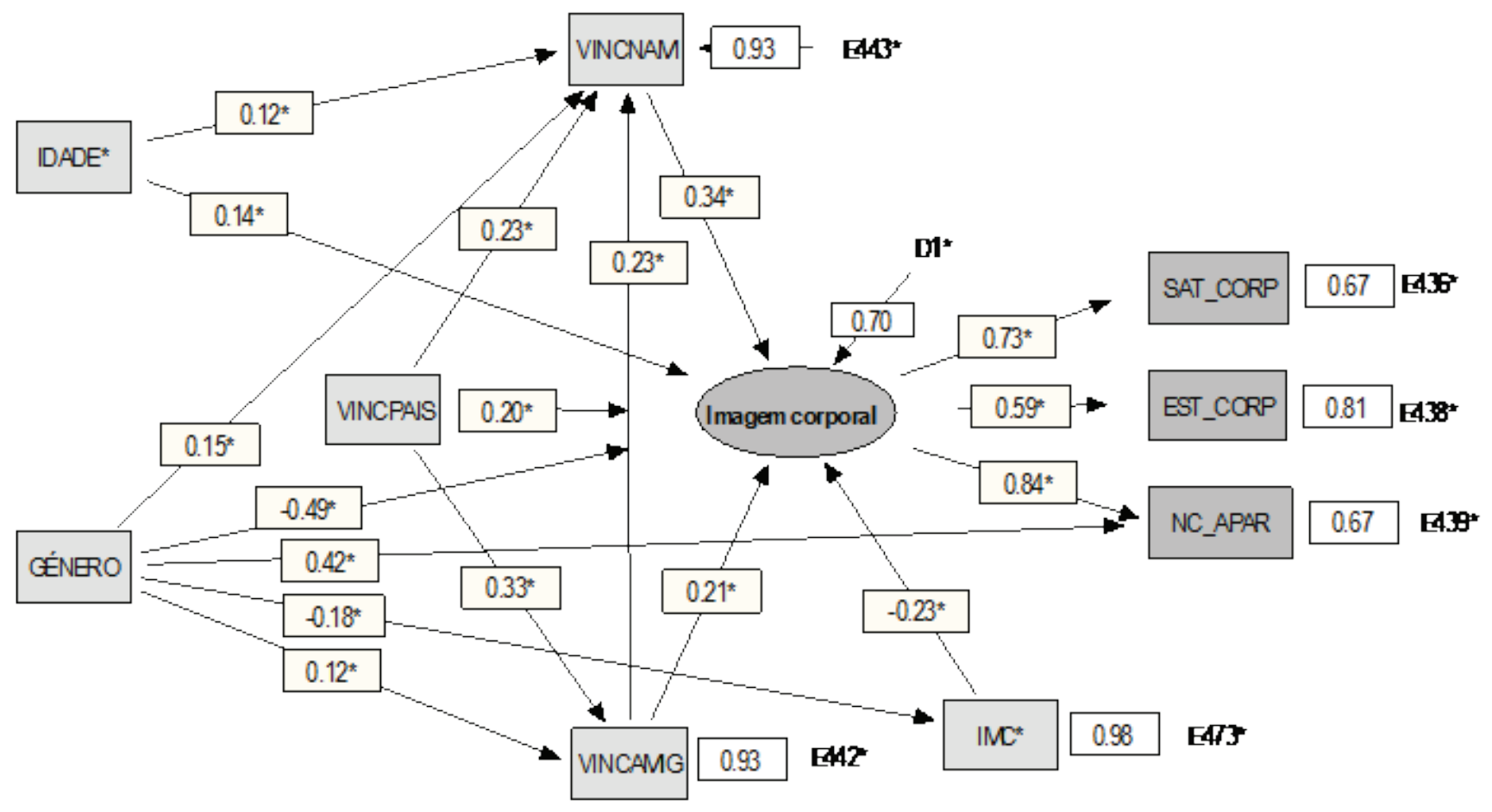

Figura 1. Modelo revisto da imagem corporal e vinculação interpessoal, género, idade e IMC.

(e validade) das medidas compostas (Rowe, 2002). Estas novas dimensões foram utilizadas nas análises seguintes.

Com base na literatura, foi proposto e testado um modelo de interrelações entre a vinculação aos pais, ao par amoroso e aos amigos, o sexo, a idade, o índice de massa corporal (IMC) e a imagem corporal. As vinculações ao pai e à mãe foram incluídas numa medida composta (vinculação parental) devido à correlação elevada entre as duas variáveis $(r=0,80)$. A variável sexo foi transformada numa variável dummy $(0=$ masculino e $1=$ feminino).

Os dados não cumprem o requisito da normalidade pelo que foram utilizados os índices robustos de adequabilidade do ajustamento; S-B $\chi^{2}$, CFI e RMSEA. O modelo teórico inicialmente proposto obteve um S-B $\chi^{2}(19,690)=215,62$, $p=0,000 ; \mathrm{CFI}=0,79$ e RMSEA $=0,12$ (IC 90\%: 0,11, 0,14). Como pode-se verificar, foram encontrados níveis abaixo do considerado razoável em termos de ajustamento global. Além disso, foram encontrados também valores muito baixos ao nível do ajustamento local, nos caminhos (paths) entre algumas das variáveis, mais precisamente entre a variável idade e a vinculação aos pais $(r=0,05)$ ou a vinculação aos amigos $(r=0,01)$, e a variável sexo e a vinculação aos pais $(r=0,08)$. Por este motivo, e porque estas situações podem ser justificadas teoricamente, optou-se por eliminar a relação entre as variáveis acima mencionadas. Com base na análise do Lagrange Multiplier test, na inspeção dos índices de modificação do modelo, verificou-se que a variável sexo estava relacionado com as variáveis Não centralidade da aparência (decréscimo esperado de 0,41 no $\chi^{2}, p<0,001$ ) e IMC (decréscimo esperado de 0,34 no $\chi^{2}, p<0,001$ ).

Após estas reespecificações, obteve-se um modelo, denominado de Modelo Revisto, com os seguintes índices: $\mathrm{S}-\mathrm{B} \chi^{2}(16,690)=40,12, \mathrm{p}=0,0007 ; \mathrm{CFI}=0,97$; e RMSEA
$=0,05$ (IC 90\%: 0,03; 0,06). Pela análise do ajustamento global verificou-se que houve um incremento no ajustamento e parcimônia do modelo. Quanto aos parâmetros individuais, todos saturaram no sentido postulado, embora com uma magnitude moderada ou baixa. Os valores de $t$ foram todos estatisticamente significativos. O modelo obtido, com estimativas dos parâmetros significativos (estandardizados), é apresentado sob a forma de diagrama na Figura 1.

De acordo com este modelo final, verificou-se que $52 \%$ da variância do constructo imagem corporal é explicado pelos seus preditores, o que é bastante razoável.

Observando as interrelações obtidas na Figura 1, e tal como se esperava, foi encontrada uma relação significativa e positiva entre a vinculação aos mais significativos e a imagem corporal, demonstrando-se nesta amostra que a imagem corporal é explicada pela qualidade da relação romântica $(\beta=0,34)$, pela relação com os pares $(\beta=0,21)$ e com os pais $(\beta=0,20)$, embora seja a relação amorosa a que mais contribui diretamente para a relação que os adolescentes têm com o seu corpo. Verificou-se ainda que a imagem corporal varia em função do gênero, da idade e do IMC (ainda que estas últimas tenham apenas um efeito reduzido), sendo as adolescentes, como se esperava, as que detêm uma imagem corporal mais negativa, comparativamente aos rapazes $(\beta=-0,49)$. De salientar que esta foi a relação mais elevada encontrada neste modelo. $\mathrm{O}$ gênero foi ainda moderadamente associado à não centralidade da aparência, mas desta vez com uma relação positiva, isto é, confirmando os resultados encontrados em análises anteriores, os rapazes são mais centrados na aparência, mais impulsivos e perfeccionistas do que as moças. Quanto ao IMC, como seria esperado, apresenta uma associação negativa $(\beta=-0,23)$ com a imagem corporal, isto é, quanto 
maior a tendência para ter peso corporal a mais, menor a satisfação com a imagem corporal. Por último, verificou-se que os adolescentes mais velhos manifestam uma imagem corporal mais positiva do que os mais novos $(\beta=0,14)$. De fato, a idade parece não ser um forte preditor, nem da imagem corporal nem da qualidade das relações mais significativas na adolescência, apresentando apenas uma associação baixa com a vinculação romântica $(\beta=0,12)$, sendo que os mais velhos apresentaram relações românticas mais positivas do que os mais novos.

Foram realizadas diversas análises complementares para clarificar as relações obtidas e todas as condições necessárias para a mediação foram cumpridas (Baron \& Kenny, 1986).

Efectivamente, um dos efeitos de mediação esperados diz respeito à relação de vinculação aos pais e a imagem corporal, mediada pela relação romântica e de amizade, em que observamos um coeficiente estandardizado para o efeito indireto de $0,15(e p=0,02, Z=6,23 \text { e } p<0,05)^{4}$. De fato, através da análise dos efeitos indiretos, verificou-se que a relação entre a vinculação aos pais e a imagem corporal é mediada pela vinculação ao namorado(a) e pela relação de amizade, parecendo indicar que a contribuição que a relação parental tem na construção de uma imagem corporal positiva na adolescência passa também pela influência que os pais podem ter no estabelecimento de relações de qualidade com os pares na vida dos filhos. Embora se verifique que a maioria do efeito da relação parental e a imagem corporal se faça de forma direta (efeito direto de 0,20, isto é, 57,4\%), 42,6\% deste efeito é realizado indiretamente, através das relações com os pares (par amoroso e amigos).

Com vista a decompor esta mediação e averiguar qual dos efeitos indiretos é o mais influente (relações de amizade ou relação amorosa), foi retirado um dos mediadores do modelo e analisado o seu impacto. Assim, retirando-se a vinculação amorosa do modelo observou-se que $24,3 \%$ do efeito da vinculação aos pais na imagem corporal é mediado pela vinculação aos amigos (coeficiente estandardizado $=0,085, e p=0,017, Z=4,78$ e $p<0,05)$. Por outro lado, removendo-se a vinculação aos amigos do modelo, verificou-se que $26,6 \%$ do efeito da vinculação aos pais na imagem corporal é mediado pela vinculação romântica (coeficiente estandardizado de 0,098, ep=0,018, $Z=4,88$ e $p<0,05)$. Constatou-se, desta forma, que ambos os mediadores, ainda que parciais, são muito equivalentes $( \pm 25 \%)$ na influência que exercem sobre o efeito da relação parental na imagem corporal. Ressalta-se também o fato de $27,7 \%$ dos efeitos da relação entre a qualidade das relações de amizade e a imagem corporal ser efetuada indiretamente, através da relação amorosa.

Concluindo, as variáveis com maior impacto (direto e indireto) na satisfação corporal são o gênero (efeito total de -0,37), a vinculação aos pais (efeito total de 0,34 ) e a vinculação ao namorado(a) (efeito total de 0,34). Finalmente, todas estas variáveis, conjuntamente com a relação com os pares, o IMC e a idade explicam $52 \%\left(R^{2}=0,521\right)$ da variância do construto Imagem corporal.

4 O EQS, tal com o outros softwares estatísticos, providencia as medidas e testes de significância dos efeitos indiretos (teste de Sobel), representado pelo erro padrão (ep) e respectivos valores de $Z$.

\section{Discussão}

No presente momento do estudo foi testado um modelo preditivo entre a vinculação aos pais, pares e par romântico e a imagem corporal, para construir um modelo global explicativo da imagem corporal que considerasse todas as variáveis analisadas no seu conjunto e as suas relações diretas e indiretas. De uma forma geral, de acordo com o modelo final encontrado, uma imagem corporal mais positiva é associada a uma melhor qualidade das relações interpessoais na adolescência, condizente com resultados de estudos prévios.

Assim, relativamente à primeira questão de investigação colocada neste estudo, acerca dos melhores preditores da imagem corporal, entre aqueles que foram considerados neste estudo, as análises revelaram que, em geral, o gênero é o preditor direto mais importante, seguido pela vinculação aos pais, a vinculação romântica e a relação de amizade. A influência combinada destes múltiplos agentes apoia a ideia de que a imagem corporal emerge num contexto diverso de forças, sublinhando-se a importância de se ter em conta todos estes e outros aspectos do estudo.

$\mathrm{Na}$ realidade foi observado um efeito positivo da qualidade das relações interpessoais mais significativas na experiência corporal, reforçando o papel central das figuras de vinculação (essencialmente a romântica) neste estádio de desenvolvimento, o que é consistente com outros estudos (Cash \& Fleming, 2004; Cash, Phillips Santos \& Hrabosky, 2004; Domini \& cols., 2000; Gerner \& Wilson, 2005; Lieberman \& cols., 2001). Efetivamente, uma vez que a construção da imagem corporal se desenvolve no contexto das relações que o indivíduo vai construindo ao longo da sua vida, na adolescência as relações amorosas terão um papel primordial, podendo ampliar ou, por outro lado desconfirmar experiências de rejeição vividas anteriormente com figuras de vinculação significativas (Matos, 2006; Matos \& Costa, 2006). Por outro lado, dada a sua natureza mais íntima, podem favorecer ou dificultar a construção de uma imagem corporal saudável e positiva. Além disso, porque o corpo é percebido como um importante componente de atratividade, não parece surpreendente que os adolescentes acreditem que a imagem corporal seja particularmente influente na obtenção de relações de sucesso com o gênero oposto e, portanto o corpo a ser vivenciado não é só como um importante produto, mas também co-construtor das próprias relações românticas.

Reforça-se também que os adolescentes com relações de vinculação inseguras com as suas figuras significativas têm igualmente uma relação mais negativa com o seu corpo. $\mathrm{Na}$ verdade, os indivíduos com menor qualidade nas suas relações tendem a centrar-se mais no seu corpo, é natural que estejam bastante condicionados à procura de aceitação pelos outros e sensíveis aos ideais sociais impostos, assimilando essas mensagens e esforçando-se ao máximo para alcançarem esse "corpo-ideal irrealista" e, consequentemente, o apreço e atenção dos outros apoiando a hipótese de que o apoio e a proximidade emocional destes agentes interpessoais, nomeadamente o encorajamento da autonomia, uma relação afectiva e carinhosa em termos físicos, além de uma comunicação aberta no contexto parental e uma relação de confiança e de segurança no contexto de pares, parecem constituir ingredientes fundamentais ao desenvolvimento de representações positivas acerca de si próprio e do seu corpo. 
Quanto à hipótese de mediação entre a qualidade da relação parental e a imagem corporal através das relações de pares (amizade e romântica), foram corroborados os resultados de outros estudos que encontraram os pares como mediadores subculturais das preocupações com o peso e corpo (Pike, 1995). Assim, embora se tenha observado que a maioria do efeito da vinculação aos pais e a imagem corporal seja direto, verificou-se um efeito de mediação parcial das relações com os pares, que se faz de forma similar pela relação dos adolescentes com os seus amigos e namorado(a). Salienta-se, deste modo, a importância da família na construção de uma vivência corporal positiva na adolescência, revelando-se a variável com mais peso total na imagem corporal seguida pelo gênero. Além disso, a qualidade do relacionamento familiar, para além de ser uma importante fonte de proteção sobre a qualidade da relação com o próprio corpo, será também uma variável central na natureza da relação que os adolescentes estabelecem com os pares e companheiros amorosos, o que é consonante com a teoria da vinculação e com a investigação empírica (ex. Ávila, Cabral \& Matos, 2008; Rocha, 2008; Rocha \& Matos, 2007; Matos, 2002).

Considera-se relevante o fato do contexto de pares (relação romântica e relação de amizade), no seu conjunto, ser responsável por $31,4 \%$ dos efeitos na imagem corporal, o que vem evidenciar o papel que este desempenha na adolescência.

Relativamente aos efeitos do gênero em cada uma das variáveis analisadas, na generalidade os resultados apoiam a hipótese formulada e corroboram a grande maioria da investigação, indicando que as mulheres ainda continuam a apresentar níveis de insatisfação com o seu corpo mais elevados do que os homens (Cash, Phillips, Santos \& Hrabosky, 2004; Barbosa, 2001; Barbosa \& Costa, 2001/2002, 2003; Green \& Pritchard, 2003; Ricciardelli \& McCabe, 2003). Assim, as mulheres que não se sentem bem com o seu corpo parecem investir numa atenção redobrada no seu físico e experienciam com mais ansiedade as suas relações, devido à importância da aparência para as relações sociais (Cash \& Fleming, 2004; McKinley \& Randa, 2005). No entanto, também como esperado, a associação observada neste estudo entre gênero masculino e a centralidade da aparência é positiva e de magnitude moderada $(r=0,42)$, o que nos indica que além dos rapazes serem mais exigentes com eles próprios (mais perfeccionistas) e mais impulsivos, já é observável que os rapazes começam a preocupar-se com a importância que a aparência pode desempenhar nas suas vidas e na relação com os outros, mesmo mais do que as adolescentes, preocupando-se mais com o seu corpo, investindo e cuidando dele. Aliás a figura masculina carece de um aprofundamento na investigação futura, nomeadamente ao nível da construção de instrumentos específicos de avaliação da imagem corporal nos rapazes.

O índice de massa corporal ou Índice de Quetelet, dado pelo peso $(\mathrm{Kg})$ /altura2, demonstrou ser um preditor importante da imagem corporal, o que já tem sido salientado em algumas pesquisas (e.g. Markey \& Markey, 2005). Os resultados sustentaram a hipótese formulada de que os participantes com mais peso teriam uma imagem corporal mais negativa do que os mais magros. Estes dados são facilmente explicados pela incorporação de crenças culturais em relação à magreza ou não-sobrepeso, como o equivalente do corpo belo, o que resulta numa visão negativa do próprio corpo. De fato, este padrão de beleza, essencialmente feminino, estimula muitos adolescentes a considerarem o seu peso ideal dentro dos limites do peso inferior ao seu, exercendo efeitos ao nível dos seus comportamentos e hábitos alimentares.

Por último, a idade, como previsto, não está fortemente associada a nenhuma variável, apresentando, contudo, uma relação com a imagem corporal e com a qualidade das relações amorosas na adolescência, reforçando os resultados encontrados neste estudo relativamente aos efeitos do grupo etário na imagem corporal (com os mais velhos a perceberem a sua imagem corporal de forma mais positiva do que os adolescentes mais novos). Na realidade, confirmando outros estudos, as preocupações corporais começam a ser cada vez mais normativas, independentes da faixa etária (Barbosa, 2001; Barbosa \& Costa, 2001/2002, 2003). Uma outra explicação para este reduzido efeito da idade, poderá ser a eventual ligação indireta à imagem corporal, através, por exemplo da internalização dos estereótipos socioculturais da aparência e beleza físicas ou da comparação social com as imagens dos mídia o que seria interessante explorar em estudos futuros, nomeadamente na próxima abordagem deste estudo.

Assim, será importante dar-se continuidade, em futura investigação, ao estudo da influência positiva que a qualidade destes contextos próximos de vinculação (pais, pares e par romântico) tem na experiência corporal, como fator ambiental protetor para aqueles adolescentes que são mais vulneráveis. No entanto, embora estes resultados tenham sido analisados, particularmente, a partir da influência que os contextos de desenvolvimento poderiam ter na construção da imagem corporal ao longo da vida, acredita-se que as relações sociais e afetivas não só influenciam a forma como o corpo é experienciado, como elas próprias são moldadas pelas próprias experiências corporais.

Finalmente, destaca-se que este é um domínio de estudo cada vez mais relevante face ao crescente interesse e preocupação sociais com questões relacionadas com o corpo, essencialmente na adolescência. Compreendendo melhor as influências interpessoais e o modo como estas interagem na forma como as pessoas se sentem com o seu próprio corpo, poderão ser providenciadas e planejadas avaliações e intervenções mais eficazes e realistas. Salienta-se assim, uma abordagem ecológica de prevenção, focalizada na mudança destes contextos assim como a relação dos participantes com eles.

\section{Referências}

Archibald, A. B. Graber, J. A., \& Brooks-Gunn, J. (1999). Associations among parent-adolescents relationships, pubertal growth, dieting and body image in young adolescents girls: A short-term longitudinal study. Journal of Research on Adolescence, 9, 395-415.

Armsden, G. C., \& Greenberg, M. T. (1987). The Inventory of Parent and Peer Attachment: Individual differences and their relationship to psychological well-being in adolescence. Journal of Youth and Adolescence, 16, 427-455.

Armstrong, J., \& Roth, D. M. (1989). Attachment and separation difficulties in eating disorders: A preliminary investigation. International Journal of Eating Disorders, 8, 141-155. 
Ávila, M., Cabral, J., \& Matos, P. M. (2008). Parental attachment and romantic relationships: the mediating role of emotion regulation and identity. XI Conference of the European Association of Research on Adolescence. Turim, Itália.

Barbosa, M. R. (2001). A influência da vinculação aos pais na imagem corporal de adolescentes e jovens. Dissertação de Mestrado, Universidade do Porto, Porto.

Barbosa, M. R., \& Costa, M.E. (2001/2002). A influência da vinculação aos pais na imagem corporal de adolescentes e jovens. Cadernos de Consulta Psicológica, 17/18, 83-94.

Barbosa, M. R., \& Costa, M. E. (2003). Body image and attachment to parents in Portuguese adolescents. In E. Palacio-Quintin, J.-M. Bouchard \& B. Terrisse (Eds.), Questions d'éducation familiale dans les années 2000 (pp.177-192). Montréal: Les Éditions Logiques.

Baron, R. M., \& Kenny, D. A. (1986). The moderator-mediator distinction in social psychological research: Conceptual, strategic and statistical considerations. Journal of Personality and Social Psychology, 51, 1173-1182.

Bartholomew, K. (1990). Avoidance of intimacy: An attachment perspective. Journal of Social and Personal Relationships, 7, 147-178.

Bartholomew, K., \& Horowitz, L. (1991). Attachment styles among young adults: A test of a four-category model. Journal of Personality and Social Psychology, 61, 226-244.

Benedikt, R., Wertheim, E. H., \& Love, A. (1998). Eating attitudes and weight-loss attempts in female adolescents and their mothers. Journal of Youth and Adolescence, 27, 43-57.

Brennan, K. A., \& Shaver, P. R. (1995). Dimensions of adult attachment, affect regulation, and romantic relationship functioning. Personality and Social Psychology Bulletin, 21, 267-283.

Broberg, A. G., Hjalmers, I., \& Nevonen, L. (2001). Eating disorders, attachment and interpersonal difficulties: A comparison between 18-to-24 year-old patients and normal controls. European Eating Disorders Review, 9, 381-396.

Bruchon-Schweitzer, M. (1990). Une psychologie du corps. Paris: Presses Universitaires de France.

Cash, T. F. (2004). Body image: Past, present, and future. Body Image: An International Journal of Research, 1, 1-5.

Cash, T. F., \& Fleming, E. C. (2004). Body image and social relations. In T. F. Cash \& T. Pruzinsky (Eds.), Body image: $A$ handbook of theory, research, and clinical practice (pp.277286). New York: Guilford Press.

Cash, T. F., \& Labarge, A. (1996). Development of the appearance schemas inventory: A new cognitive body-image assessment. Cognitive Therapy and Research, 20, 37-50.

Cash, T., \& Pruzinsky, T. (1990). Body Images. Development, Deviance and Change. NY and London: The Guilford Press.

Cash, T. \& Pruzinsky, T. (2004). Body Image. A Handbook of theory, research \& clinical practice. NY and London: The Guilford Press.

Cash, T. F., Phillips, K. A., Santos, M. T., \& Hrabosky, J. I. (2004). Measuring "negative body image": validation of the Body Image Disturbance Questionnaire in a nonclinical population. Body image, 1, 363-372.

Cash, T. F., Theriault, J., \& Annis, N. W. (2003). Body image in an interpersonal context: Adult attachment, fear of intimacy, and social anxiety. Journal of Social and Clinical Psychology, 23, 89-103.
Demarest, J., \& Allen, R. (2000). Body Image: Gender, ethnic, and age differences. Journal of Social Psychology, 140, 465-72.

Domini, N. L., Johnson, W. B., \& Koch, K. (2000). Perception of parental acceptance in women with binge eating disorder. The Journal of Psychology, 134, 23-40.

Evans, L., \& Wertheim, E. H. (2005). Attachment styles in adult intimate relationships: Comparing women with bulimia nervosa symptoms, women with depression and women with no clinical symptoms. European Eating Disorders Review, 13, 285-293.

Franzoi, S. L., \& Shields, S. (1984). The Body Esteem Scale: Multidimensional structure and sex differences in a college population. Journal of Personality Assessment, 48, 173-178.

Garner, D. M. (1991). Eating Disorder Inventory-2. Professional Manual. USA: Psychological Assessment Resources, Inc.

Gaspar, N. M. (1999). Estudo da Imagem Corporal na Adolescência. Dissertação de Mestrado, Universidade de Coimbra, Coimbra.

Gerner, B., \& Wilson, P. H. (2005). The relationship between friendship factors and adolescent girls' body image concern, body dissatisfaction, and restrained eating. International Journal of eating disorders, 37, 313-320.

Green, S. P., \& Pritchard, M. E. (2003). Predicting body image dissatisfaction in adult men and women. Social Behavior and Personality, 31, 215-222.

Grogan, S. (1999). Body Image. London: Routhledge.

Kearney-Cooke, A. (2004). Familial influences on body image development. In T. F. Cash, \& T. Pruzinsky (Eds.), Body image: A handbook of theory, research, and clinical practice (pp. 99107). New York: Guilford Press.

Lieberman, M., Gauvin, L., Bukowski, W., \& White, D. R. (2001). Interpersonal influence and disordered eating behaviours in adolescent girls: The role of peer modelling, social reinforcement, and body-related teasing. Eating Behaviours, 2, 215-236.

Lunner, K., Wertheim, E. H., Thompson, J. K., Paxton, S. J., McDonald, F., \& Halvarsson, R. (2000). A cross-cultural investigation of the role of teasing, and weight and dieting concerns in adolescent girls from Sweden and Australia. International Journal of Eating Disorders, 48, 430-435.

Markey, C. N., \& Markey, P. M. (2005). Relations between body image and dieting behaviors: An exploration of gender differences. Sex Roles: A Journal of Research, 53, 519-530.

Matos, P. M. (2002). (Des)continuidades na vinculação aos pais e ao par amoroso em adolescentes. Tese de Doutorado, Universidade do Porto, Porto.

Matos, P. M. (2006). Relações românticas em adolescentes. Psychologica, 41, 9-24.

Matos, P. M., Almeida, H. M., \& Costa (1998). Dimensions of attachment to mother and father in Portuguese adolescents. Poster presented on 6th Biennial Conference of the European Association for Research on Adolescence, Budapest, Hungria.

Matos, P. M., Barbosa, S., \& Costa, M. E. (1998). Avaliação da vinculação amorosa em adolescentes e jovens adultos: Construção de um instrumento e estudos exploratórios. Comunicação apresentada na Conferência Internacional de Avaliação Psicológica, Formas e Contextos. Salamanca, Espanha. 
Matos, P. M., Barbosa, S., \& Costa, M. E. (1999). Representations of attachment to parents and to romantic partners in Portuguese late adolescents. Comunicação apresentada no VI Congresso Europeu de Psicologia, Roma, Itália.

Matos, P. M. \& Costa, M. E. (2006). Vinculação aos pais e ao par romântico em adolescentes. Psicologia, 20(1), 97-126.

McCabe, M. P., \& Ricciardelli, L. A. (2001). Body image and body change techniques among young adolescent boys. European Eating Disorders Review, 9, 335-347.

McKinley, N. M., \& Randa, L. A. (2005). Adult attachment and body satisfaction. An exploration of general and specific relationship differences. Body Image, 2, 209-218.

Muth, J. L., \& Cash, T. F. (1997). Body-image attitudes: What differences does gender make? Journal of Applied Social Psychology, 27, 1438-1452.

O'Dea, J., \& Abraham, S. (1999). Association between self-concept and body weight, gender, and pubertal development among male and female adolescents. Adolescence, 34, 69-79.

Okine, K. N. (2000). The relationship aspects of eating disorder symptomatology. Dissertation Abstracts International, 61, 32-87.

Pike, K. M. (1995). Bulimic symptomatology in high school girls. Psychology of Women Quarterly, 19, 373-396.

Ricciardelli, L. A., \& McCabe, M. P. (2003). Sociocultural and individual influences on muscle gain and weight loss strategies among adolescent boys and girls. Psychology in the Schools, 40, 209-224.

Ricciardelli, L. A., McCabe, M. P., \& Banfield, S. (2000). Sociocultural influences on body change methods in adolescent boys. Journal of Psychosomatic Research, 49, 189-197.

Rocha, M. (2008). O desenvolvimento das relações de vinculação na adolescência: associações entre contextos relacionais com pais, pares e par amoroso. Tese de Doutorado, Universidade do Porto, Porto.

Rocha, M., \& Matos, P. M. (2007). Qualidade da vinculação e adaptação escolar: qual o papel da auto-estima? XI Congresso Internacional de Educação Familiar, Universidade de Coimbra, Abril. Coimbra, Portugal.
Rowe, K. (2002). The measurement of latent and composite variables from multiple items or indicators: Applications in performance indicator systems. Background paper prepared for keynote address presented for the Royal Melbourne Institute of Technology Statistics Seminar Series.

Sharpe, T. M., Killen, J. D., Bryson, S. W., Shisslak, C. M., Estes, L. S., Gray, N., Crago, M., \& Taylor, C. B. (1998). Attachment style and weight concerns in preadolescent and adolescent girls. International Journal of Eating Disorders, 23, 39-44.

Sprecher, S., McKinney, K., \& Orbuch, T. L. (1991). The effects of current sexual behavior on friendship, dating, and marriage desirability. The Journal of Sex Research, 28, 387-408.

Stice, E., \& Whitenton, K. (2002). Risk factors for body dissatisfaction in adolescent girls: A longitudinal investigation. Developmental Psychology, 38, 669-678.

Suldo, S. \& Sandberg, D. (2000). Relationship between attachment styles and eating disorder symptomatology among college women. Journal of College Student Psychotherapy, 15, 59-73.

Tantleff-Dunn, S., \& Gokee, J. L. (2004). Interpersonal influences on body image development. In T. F. Cash \& T. Pruzinsky (Eds.), Body image: A handbook of theory, research, and clinical practice (pp. 108-116). New York: Guilford Press.

Thompson, J. K. (1996). Body image, eating disorders, and obesity: An integrative guide for assessment and treatment. Washington, DC: American Psychological Association.

Ward, A., Ramsay, R., \& Treasure, J. (2000). Attachment research and eating disorders. British Journal of Medical Psychology, $73,35-51$. 